\title{
OPERATOR ALGEBRAS AND ALGEBRAIC $K$-THEORY
}

\author{
LAWRENCE G. BROWN ${ }^{1}$
}

Communicated by I. M. Singer, June 23, 1975

1. Introduction. We wish to announce several related results which demonstrate a relationship between operator theory and algebraic $K$-theory. Some of these results concern extensions of $C^{*}$-algebras (cf. [4], [5]) and complement the results of [4]. Others concern the trace and determinant invariants defined in [7].

2. Extensions of $C^{*}$-algebras. Let $H$ be a separable infinite dimensional Hilbert space, $L(H)$ the algebra of bounded linear operators on $H, K$ the ideal of compact operators, and $A=L(H) / K$. In [4] and [5] $\operatorname{Ext}(X)$ was defined as the set of equivalence classes of $C^{*}$-algebra extensions, $0 \rightarrow K \rightarrow E \rightarrow C(X)$ $\rightarrow 0$, for $X$ a compact metric space and $C(X)$ the algebra of continuous complex functions on $X$. $\operatorname{Ext}(X)$ was also described as unitary equivalence classes of *. isomorphisms $\tau: C(X) \rightarrow A$. It was shown that $\operatorname{Ext}(X)$ is a group and that it gives rise to a generalized homology theory which is related to $K$-theory in roughly the same way as homology is related to cohomology. A Bott periodicity map, Per: $\operatorname{Ext}\left(S^{2} X\right) \rightarrow \operatorname{Ext}(X)$, was defined and was proved to be injective for all $X$ and surjective for smooth $X$. Also $\operatorname{Ext}(X)$ was given the structure of a not necessarily Hausdorff topological group, and the closure of the identity was called $\operatorname{PExt}(X)$.

THEOREM 1. Per is surjective for all $X$.

THEOREM 2. There is a natural short exact sequence,

$$
0 \rightarrow \operatorname{Ext}_{Z}^{1}\left(K^{0}(X), Z\right) \rightarrow \operatorname{Ext}(X) \stackrel{\gamma_{\infty}}{\rightarrow} \operatorname{Hom}\left(\widetilde{K}^{1}(X), Z\right) \rightarrow 0,
$$

which splits noncanonically.

COROllary. PExt $(X)$ is the maximum divisible subgroup of $\operatorname{Ext}(X)$.

THEOREM 3. If $\tau_{t}: C(X) \rightarrow A, 0 \leqslant t \leqslant 1$, is a continuous family in the sense that $\tau_{t}(f)$ is continuous for each $f \in C(X)$, then each $\tau_{t}$ defines the same element of $\operatorname{Ext}(X)$.

For a more leisurely account of these results, see [3]. See also [4], [5], [8]. Ext ${ }_{*}$ satisfies parallel axioms to the Steenrod homology theory [11], whose axiomatic description in [10] plays a key role in the proofs. Algebraic $K$-theory

AMS (MOS) subject classifications (1970). Primary $46 \mathrm{LO5}$.

1 Research partially supported by a grant from the National Science Foundation. 
(cf. [9]) also plays a key role by yielding a natural definition of an isomorphism $\kappa:$ ker $\gamma_{\infty} \rightarrow \operatorname{Ext}_{\mathbf{Z}}^{1}\left(K^{0}(X), Z\right) . \kappa$ is defined by applying the algebraic $K$-theory long exact sequence to $0 \rightarrow K \rightarrow E \rightarrow C(X) \rightarrow 0$ and obtaining (in part)

$$
0 \rightarrow \mathrm{Z} \cong K_{0}(K) \rightarrow K_{0}(E) \rightarrow K_{0}(C(X)) \cong K^{0}(X) \rightarrow 0 .
$$

We are grateful to J. Milnor, J. Kaminker, and C. Schochet for valuable communications.

In another context [2] we have defined an almost polonais group as the quotient of a polonais (complete, separable, metrizable) group by a normal subgroup which is a continuous homomorphic image of a polonais group. These are not necessarily Hausdorff topological groups with some additional structure, and the abelian ones form an abelian category. Theorem 2 shows that $\operatorname{Ext}(X)$ is the direct sum of two almost polonais groups, and we would like to know whether $\operatorname{Ext}(X)$ is naturally such an object.

3. The trace and determinant invariants. Let $\mathscr{A}$ be a *-sublagebra of $L(H)$ such that $\mathscr{H}$ contains the trace class, $J$, and is commutative modulo $J$. As in [7], we obtain a symbol map $\phi: \mathfrak{I} \rightarrow C(X)$. Here we assume $X \subset \mathbf{R}^{n}$ and range $\phi=$ $C^{\infty}(X)$, the algebra of restrictions to $X$ of $C^{\infty}$ functions on $\mathbf{R}^{n}$. Let $\widetilde{X}$ be a closed ball containing $X$. Helton and Howe [7] defined a trace invariant $l: \Omega \rightarrow \mathrm{C}$, where $\Omega$ is the space of exact $C^{\infty}$ 2-forms on $\widetilde{X}$ and $l(d f \wedge d g)=\operatorname{tr}(A B-B A)$, where $A$ and $B$ are elements of $\mathfrak{U}$ such that $\phi(A)=f \mid X$ and $\phi(B)=g \mid X$. If $A$ and $B$ are invertible, Helton and Howe also considered $\operatorname{det}\left(A B A^{-1} B^{-1}\right)=$ $\delta(\phi(A), \phi(B)) . \delta$ is a bimultiplicative form on a subgroup of the group of units in $C^{\infty}(X)$. In [1] we showed, in the special case $X \subset \mathbf{R}^{2}$, that $\delta$ can be extended to a form $d$ on the whole group of units and that $d$ can be calculated from the trace invariant. As suggested to us by $\mathrm{H}$. Sah, the algebraic properties of $d$ provided an analogy with algebraic $K$-theory. We will now define a new determinant invariant, $d_{1}: K_{2}\left(C^{\infty}(X)\right) \rightarrow \mathrm{C}^{*}$, such that $d$ is the restriction of $d_{1}$ to the Steinberg symbols.

Consider the short exact sequence, $0 \rightarrow J \rightarrow \mathfrak{U} \rightarrow \mathfrak{A} / J \rightarrow 0$, and the corresponding algebraic $K$-theory long exact sequence $\cdots \rightarrow K_{2}(\mathfrak{A} / J) \rightarrow$ $K_{1}(J) \rightarrow K_{1}(\mathfrak{U}) \cdots$. Using the definition of $K_{1}(J)$ and the most basic properties of the determinant (on the determinant class, $I+J \subset L(H)$ ), we obtain a map det: $K_{1}(J) \rightarrow \mathrm{C}^{*}$. This pulls back to $d^{\prime}: K_{2}(\mathscr{U} / J) \rightarrow \mathrm{C}^{*}$. Using analytic techniques (mainly suggested by [7]), we can modify $d^{\prime}$ to obtain $d_{1}$.

The restriction of $d_{1}$ to $K_{2}^{\prime}$, the range of $K_{2}\left(C^{\infty}(\widetilde{X})\right) \rightarrow K_{2}\left(C^{\infty}(X)\right)$ (which is the same as the kernel of $\left.K_{2}\left(C^{\infty}(X)\right) \rightarrow K^{2}(X)\right)$, can be calculated from the trace invariant: Roughly one shrinks $\widetilde{X}$ to a point and differentiates with respect to "time". In this way we obtain a map $\theta: K_{2}\left(C^{\infty}(\widetilde{X})\right) \rightarrow \Omega$, and $d_{1}(C)=\exp (l(\theta(\widetilde{C})))$, for $\widetilde{C} \in K_{2}\left(C^{\infty}(\widetilde{X})\right)$ and $C$ its image in $K_{2}\left(C^{\infty}(X)\right)$. The above leads to an explicit formula for $\theta$. See [6] for the relation and application of this formula to algebraic $K$-theory. Although $\widetilde{C}$ is not uniquely determined by 
$C$, the restriction of $\theta(\widetilde{C})$ to $X$ is unique. If $l$ vanishes at 2-forms which vanish on $X$, then we obtain $l^{\prime}: K_{2}^{\prime} \rightarrow C$. According to [7], this occurs precisely when $\overline{\mathfrak{A}}$ is an element of ker $\gamma_{\infty} \subset \operatorname{Ext}(X)$, and one can then ask whether $l^{\prime}$ can be extended to $l^{\prime \prime}: K_{2}\left(C^{\infty}(X)\right) \rightarrow \mathrm{C}$ such that $d_{1}=\exp \left(l^{\prime \prime}\right)$. This leads to an element of $\operatorname{Ext}_{Z}^{1}\left(K^{0}(X), Z\right)$, which vanishes precisely when $l^{\prime \prime}$ exists.

REMARKS 1. Although the construction just completed motivated $\kappa$, we do not know whether the two constructions actually agree.

2. The algebra $\mathscr{I}$ is what Helton and Howe call a "one dimensional" algebra. It would be nice to extend the above to the " $k$-dimensional" algebras of [7]. There seem to be two difficulties: (a) So far as we know, no existing treatment of $K_{n}$ for $n>2$ lends itself to explicit formulas as well as [9]. (b) In the $k$-dimensional case the determinant invariant ought to be defined on $K_{2 k}$; but if we do what is natural in the context of [7], we get something on $K_{k+2}$ (for $k>1$ ). Thus perhaps something is wrong for $k>2$.

We hope that these difficulties will eventually be surmounted and that the result will be significant mutual enrichment of operator theory and algebraic $K$-theory.

\section{REFERENCES}

1. L. G. Brown, The determinant invariant for operators with compact self-commutators, Proc. Conf. on Operator Theory, Lecture Notes in Math., vol. 345, Springer-Verlag, New York, 1973, pp. 210-228.

2. Group cohomology of topological groups (in preparation).

3. - Characterizing $\operatorname{Ext}(X)$, Lecture at Internat. Conf. on $K$-theory and Operator Algebras (Athens, Georgia, April, 1975), Lecture Notes in Math., Springer-Verlag, New York (to appear).

4. L. G. Brown, R. G. Douglas and P. A. Fillmore, Extensions of $C^{*}$-algebras, operators with compact self-commutators, and K-homology, Bull. Amer. Math. Soc. 79 (1973), 973-978.

5. Unitary equivalence modulo the compact operators and extensions of $C^{*}$-algebras, Proc. Conf. on Operator Theory, Lecture Notes in Math., vol. 345, SpringerVerlag, New York, 1973, pp. 58-128.

6. R. K. Dennis, Differentials in algebraic K-theory (preprint).

7. J. W. Helton and R. E. Howe, Integral operators: commutators, traces, index and homology, Proc. Conf. on Operator Theory, Lecture Notes in Math., vol. 345, SpringerVerlag, New York, 1973, pp. 141-209.

8. J. Kaminker and C. Schochet, Steenrod homology and operator algebras, Bull. Amer. Math. Soc. 81 (1975), $431-434$.

9. J. W. Milnor, Introduction to algebraic K-theory, Ann. of Math. Studies, no. 72, Princeton, N. J., 1971.

10. J. W. Milnor, On the Steenrod homology theory, Mimeographed notes, Univ. of Calif, Berkeley, Calif., 1961.

11. N. Steenrod, Regular cycles on compact metric spaces, Ann. of Math. (2) 41 (1940), 833-851. MR 2, 73.

DEPARTMENT OF MATHEMATICS, PURDUE UNIVERSITY, WEST LAFAYETTE, INDIANA 47907 